\title{
Colorectal malignant polyps: a modern approach
}

\author{
Sofia Saraiva ${ }^{a}$ Isadora Rosa ${ }^{a}$, Ricardo Fonseca ${ }^{b}$, António Dias Pereira ${ }^{a}$
}

Instituto Português de Oncologia de Lisboa Francisco Gentil, Lisboa, Portugal

Abstract

\begin{abstract}
Colorectal malignant polyps (MP) are polyps with invasive cancer into the submucosa harboring a variable risk of lymph node involvement, which can be estimated through evaluation of morphological, endoscopic, and histologic features. The recent advances in imaging endoscopic techniques have led to the possibility of performing an optical diagnosis of T1 colorectal cancer, allowing the selection of the best therapeutic modality to optimize outcomes for the patient. When MP are diagnosed after endoscopic removal, their management can be challenging. Differentiating low- and high-risk histologic features that influence the possibility of residual tumor, the risk of recurrence and the risk of lymph node metastasis, is crucial to further optimize treatment and surveillance plans. While the presence of high-risk features indicates a need for surgery in the majority of cases, location, comorbidities and the patient's preference should be taken in account when making the final decision. This is a particularly important issue in the management of low rectal MP presenting with high-risk features, where chemoradiotherapy followed by a watchand-wait strategy has demonstrated promising results. In this review we discuss the important prognostic features of MP and the most modern approaches regarding their management.
\end{abstract}

Keywords Colorectal malignant polyp, colorectal cancer, endoscopic resection, surveillance

Ann Gastroenterol 2021; 34 (x): 1-11

\section{Introduction}

Colorectal cancer (CRC) accounts for 10\% of all tumor types worldwide; it is the third most common cancer in males and the second in females [1,2]. In developing countries, there has been an increasing incidence of CRC, while in developed countries, a stabilization or a decrease in incidence is being observed, along with a decline in mortality due to successes in prevention and improved treatment options [2-4].

Most CRC arise from "classical" adenomatous polyps, through the traditional adenoma-carcinoma sequence, mostly via the well characterized chromosomal instability pathway $[1,5,6]$. More recently, a distinct molecular pathway has been described: the serrated neoplasia pathway, accounting

${ }^{a}$ Gastroenterology Department (Sofia Saraiva, Isadora Rosa, António

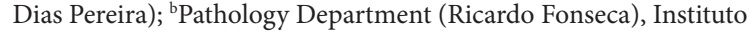
Português de Oncologia de Lisboa Francisco Gentil, Lisboa, Portugal

Conflict of Interest: Isadora Rosa reports grants, personal fees, or nonfinancial support from AbbVie, Ferring, MSD, Takeda, Pharmakern, Janssen, and Dr Falk Pharma, outside the submitted work. The remaining authors have nothing to disclose

Correspondence to: Sofia Saraiva, Serviço de Gastrenterologia, Pavilhão Central Piso 3, Rua Professor Lima Basto, Pavilhão Central, 1099-023

Lisbon, Portugal, e-mail: asofia.saraiva@gmail.com

Received 24 July 2021; accepted 2 November 2021; published online 6 December 2021

DOI: https://doi.org/10.20524/aog.2021.0681 for approximately $30 \%$ of CRC. In this critical route to CRC, the adenoma-carcinoma sequence is accelerated, and sessile serrated lesions are the main precursor lesions [7].

CRC screening programs have led to improvements in the detection of early CRC and to an increase in the number of people identified as having malignant polyps (MP) $[2,8,9]$. The term MP refers to polyps whose histology confirms the presence of carcinoma invading through the muscularis mucosae into the submucosa without penetrating the muscularis propria, regardless of lymph node involvement $[8,10,11]$. These lesions are classified as pT1 in the TNM classification system $[4,8]$. After endoscopic removal of polyps, the prevalence of MP varies between $0.75 \%$ and $5.6 \%$, constituting $40-60 \%$ of stage I CRC $[6,9,12]$. The potential for lymph node metastasis (LNM) associated with MP varies according to a combination of risk features, namely depth of invasion. Thus, polyps with characteristics suggestive of superficial invasion, such as pedunculated polyps Haggitt level 1 or sessile polyps Kikuchi sm1, are associated with an almost negligible risk of LNM $(0-3 \%)$. On the other hand, the risk of LNM may be as high as $25 \%$ for pedunculated Haggitt 4 or sessile polyps $[5,13]$. The knowledge of identifiable adverse histologic features, the advances in imaging techniques that allow for optical diagnosis of T1 CRC and the recent advances in endoscopic treatment techniques have led to an increasing awareness of the importance of MP [5,14-16]. The purpose of this article is to review recent data on the histopathology and endoscopic features of MP, to discuss the various management strategies available, and to review evidence concerning the optimal 
selection of patients for endoscopic surveillance vs. surgical treatment after endoscopic resection of MP.

\section{Optical diagnosis of T1 CRC}

During colonoscopy, various histological subtypes of colorectal polyps can be identified, including serrated and adenomatous lesions and MP. All colorectal lesions should undergo careful and complete endoscopic mucosal inspection to accurately differentiate between adenomas and CRC and to predict the depth of invasion $[8,17]$. Deep submucosal invasion (SMI) refers to an invasion depth $>1000 \mu \mathrm{m}$ and is associated with a high risk of residual cancer and LNM $[8,18]$.

When performing endoscopic evaluation of colorectal lesions, there are specific endoscopic features that need to be assessed. Some include gross morphological aspects assessed by white-light colonoscopy and others involve image-enhanced endoscopy techniques that use dye or equipment to improve visualization of lesions $[15,19]$. According to recent evidence, the use of the advanced imaging techniques was considered preferential to the use of gross morphological features for the optical diagnosis of T1 CRC and deep SMI [16].

\section{White light characterization}

Gross morphological features include morphology, size and location. Although the sensitivity ranges from 0.18-0.68 and the specificity from $0.80-0.98$ [16], gross morphological features should still be inspected carefully during endoscopy.

\section{Morphology and surface characteristics}

Endoscopic classification of superficial colorectal lesions should follow the Paris classification, which stratifies neoplasia macroscopically into polypoid (pedunculated 0-Ip and sessile 0 -Is), nonpolypoid (elevated 0-IIa, flat 0-IIb, depressed 0-IIc), and excavated or ulcerated lesions (0-III) [18]. The risk of invasive CRC can be estimated from each lesion's size and Paris classification (Table 1) [14-21].

The category missing from the Paris Classification is the lateral spreading tumor (LST). These are lesions that are $>10 \mathrm{~mm}$, with flat (0-II), or sessile (0-Is) morphology, and present lateral extension (rather than vertical) along the colonic wall. According to the Kudo classification, LSTs are further classified into granular (LST-G), which includes the homogeneous and nodular mixed subtypes, and non-granular (LST-NG), which comprises the flat elevated and pseudodepressed subtypes [8,21]. LSTs harbor a higher risk of invasive cancer. A recent systematic review and meta-analysis, which included 48 studies, found that the risk of SMI is associated with the endoscopic Kudo subtype, the lesion's size and its location. SMI is most common in pseudo-depressed nongranular LSTs $(31.6 \%)$ (Fig. 1) and nodular mixed granular LSTs (10.5\%) (Fig. 2) [22]. As such, LSTs with a higher risk of containing SMI should have their depressed and nodular areas carefully inspected using not only white-light endoscopy, but also advanced imaging techniques, as this will provide important information for treatment decisions.

\section{Size}

Previous studies have shown a correlation between a lesion's size and its potential for malignancy [23,24]. Larger lesions are at higher risk for SMI and lymph node involvement. In a prospective cohort of 2277 LST $20 \mathrm{~mm}$ by Burgess et al [25], SMI was evident in $7.6 \%$ of cases. Similarly, Bogie et al [22] found that the proportion of SMI increased with size (10-19 mm, 4.6\%; 20-29 mm, 9.2\%; $\geq 30 \mathrm{~mm}, 16.5 \%)$. However, lesion size does not have enough discriminant power to be used alone [8]. In fact, Matsuda et al [26] showed that small non-polypoid lesions are more likely to contain carcinoma and a deeper infiltration of the submucosa when compared to some larger polypoid lesions, such as homogeneous granular 0-IIa LSTs.

\section{Location}

Location is another important variable in predicting SMI and in therapeutic decision making. In the meta-analysis conducted by Bogie et al [22], SMI was more common distally, rather than when LST were proximal to the splenic flexure (odds ratio [OR] 2.50, 95\% confidence interval [CI] 1.24-5.02). This finding is related to the type of LSTs more often found in the distal colon. In fact, the same authors found that pseudodepressed non-granular and nodular mixed granular LSTs, which have a higher risk of SMI, are more often located distally.

Table 1 Risk of submucosal invasion according to Paris classification and size (adapted from [20]). Data were obtained from an endoscopy series with pathology confirmation including 19,560 lesions in the period April 1985 to April 2003. Values are given as \% (n/n total)

\begin{tabular}{lccccc}
\hline Size & $<5 \mathrm{~mm}$ & $6-10 \mathrm{~mm}$ & $11-15 \mathrm{~mm}$ & $16-20 \mathrm{~mm}$ \\
\hline $\begin{array}{l}\text { Paris } \\
\text { classification }\end{array}$ & & & & \\
\hline -I & & & & & \\
0 -Ip + 0-Is & $0 \%(0 / 5400)$ & $1.2 \%(49 / 4045)$ & $8 \%(80 / 1002)$ & $17 \%(58 / 330)$ & $30 \%(56 / 187)$ \\
0 -II & & & & & \\
0 -IIa + IIb & $<0.1 \%(2 / 6214)$ & $0.2 \%(2 / 1015)$ & $1.8 \%(9 / 493)$ & $10 \%(17 / 165)$ & $23 \%(53 / 235)$ \\
0 -IIc & $7 \%(17 / 236)$ & $44 \%(58 / 132)$ & $67 \%(42 / 63)$ & $90 \%(18 / 20)$ & $87 \%(13 / 15)$ \\
\hline
\end{tabular}




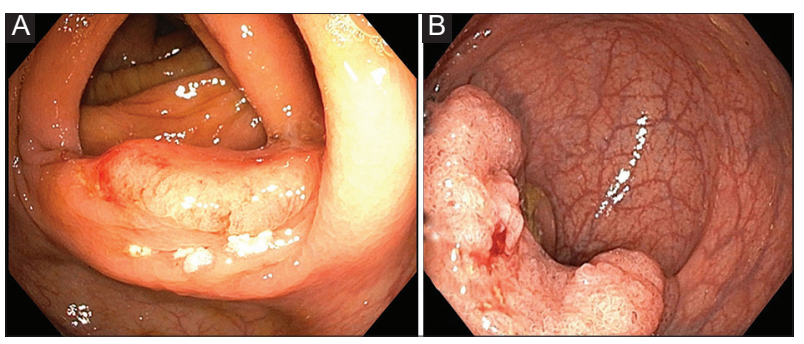

Figure 1 Pseudo-depressed non-granular lateral spreading tumor of the transverse colon $(\mathrm{A})$ and rectum $(\mathrm{B})$

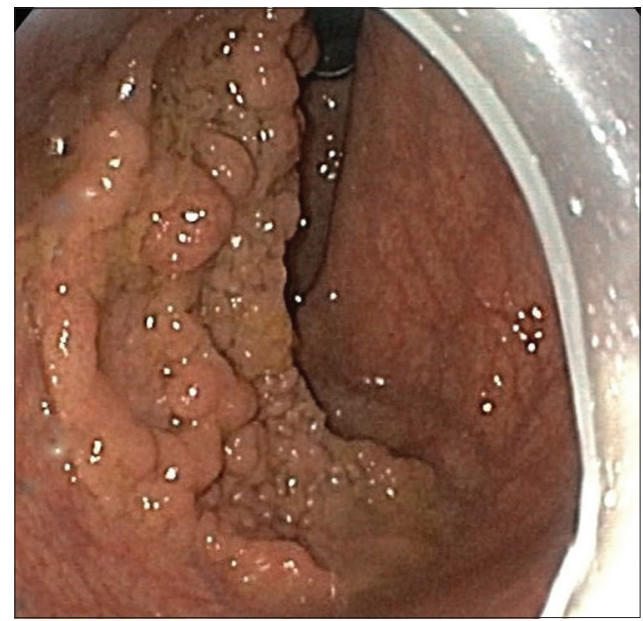

Figure 2 Nodular mixed granular lateral spreading tumor of the rectum

Burgess et al [25] also found that the frequency of SMI was significantly higher in LSTs located in the rectum or sigmoid colon $(13.1 \%)$, when compared with more proximal lesions $(5.2 \% ; \mathrm{P}<0.001)$. Within the serrated pathway, most carcinomas arise in the cecum or ascending colon, and approximately onethird arise in the rectum [7].

\section{Other gross morphological malignant features}

Studies reporting on endoscopic criteria to stratify for the risk of SMI also identified other gross morphological features that correlate with SMI. In a prospective multicenter study including more than 2000 lesions $>10 \mathrm{~mm}$, Puig et al [14] found that non-lifting, chicken-skin sign (pale yellow-speckled mucosa), edge retraction, depressed areas, fold convergence, induration, ulceration, and polyp over polyp were all significantly associated with deep SMI (>1000 $\mu \mathrm{m})$ (Fig. 3). Although these associations have been observed, the sensitivity of these features for diagnosing deep SMI seems to be low, ranging from 0.18-0.68, with specificity varying from 0.8-0.98 [24].

\section{Image enhancing techniques}

Examination of suspicious areas using white-light endoscopy should be complemented by the use of chromoendoscopy and/

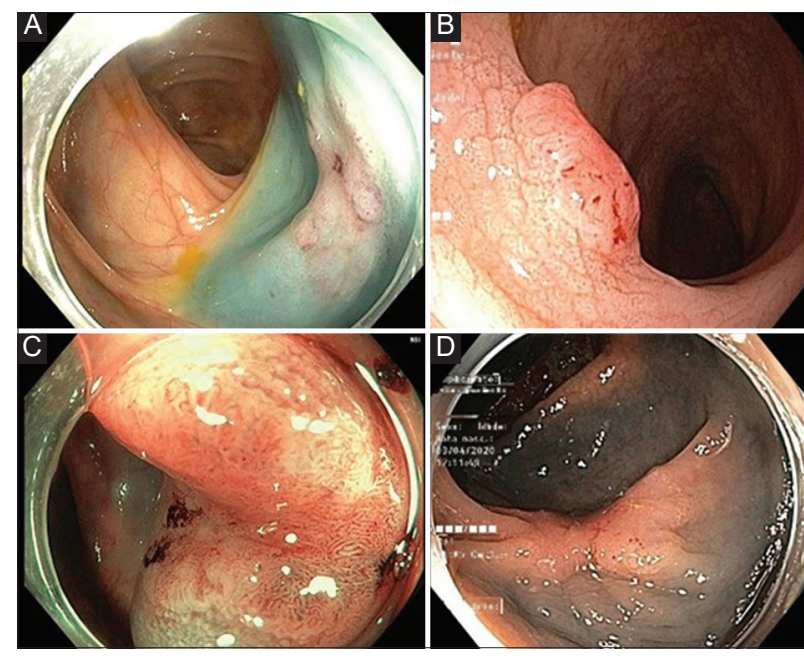

Figure 3 Examples of gross morphologic features associated with deep submucosal invasion, namely non-lifting sign (A), chicken-skin sign (B), depressed areas (C), and fold convergence (D)

or optical enhancement $[15,27]$. These techniques are useful in predicting histology, providing an "optical biopsy" [6].

Image-enhanced endoscopy, including dye-assisted conventional chromoendoscopy and virtual chromoendoscopy, with or without magnification, is continually evolving in order to improve mucosal visualization and enhance fine structural and microvascular details $[15,16,24,28]$.

Dye-assisted chromoendoscopy using indigo carmine, with or without crystal violet, is the traditional method used to emphasize the surface pattern of the colonic mucosa. Equipment-based virtual chromoendoscopy has the advantage of being relatively easy and ready to use. There are 2 types of virtual chromoendoscopy: optical chromoendoscopy and digital chromoendoscopy $[6,8,28]$. Optical chromoendoscopy technologies use optical lenses to narrow the bandwidth of spectral transmittance, thereby unveiling the mucosal and submucosal blood vessels-e.g., narrow-band imaging (NBI) (Olympus Medical Systems, Tokyo, Japan). Digital chromoendoscopy technologies are based on the digital postprocessing of acquired images, resulting in enhanced tissue contrast-e.g., flexible spectral imaging color enhancement (FICE) (Fujinon Intelligent Chromo Endoscopy; Fujifilm, Tokyo, Japan) and i-Scan digital contrast (I-SCAN) (Pentax, Tokyo, Japan) $[6,8,28]$. More recently, an endoscopic system using laser as the light source has been developed (Blue Laser Imaging [BLI]; Fujifilm, Kanagawa, Japan). By using 2 monochromatic lasers, BLI allows the production of white-light images as well as NBI, enhancing surface and vascular structures [29].

Several classifications have been created to assist with lesion characterization when using image enhancement. For microvascular pattern evaluation, the NBI international colorectal endoscopic (NICE) classification and the Japanese NBI expert team (JNET) classification are the most widely disseminated, while the Kudo classification is the gold standard for pit pattern assessment using crystal violet $[15,24]$.

The NICE classification, proposed in 2009 and internationally validated, is based on color, vessels and surface pattern of lesions 
seen on non-magnifying endoscopy. It classifies colorectal neoplasms into 3 categories: type 1 - hyperplastic lesions, type 2 - adenomas, and type 3 - invasive tumors (Fig. 4) [19,30].

The accuracy of the NICE classification for identifying lesions with deep invasion in routine clinical practice without magnification, by western endoscopists, was recently assessed by Puig et al [14]. They found this classification system to be effective, with a sensitivity of $58.4 \%$ and a specificity of $96.4 \%$, a positive predictive value of $41.6 \%$, and a negative predictive value of $98.1 \%$ for deep invasion. However, the authors stressed the importance of evaluating polyp morphology (pedunculated vs. non-pedunculated), the presence of ulceration, depressed areas or a granular LST nodular mixed-type morphology, along with the NICE classification, when evaluating colorectal lesions.

Virtual chromoendoscopy without magnification is enough for predicting or ruling out deep SMI in clinical practice for most lesions. However, magnifying virtual chromoendoscopy is needed to make an accurate diagnosis in nonulcerated NICE type 3 lesions or NICE type 2 lesions with depressed areas or of nodular mixed type. JNET classification [31] focuses on vessel and surface patterns assessed by magnification and maintains NICE types 1 and 3 but subdivides adenomatous lesions (NICE type 2) into type 2A, namely low-grade adenomas, and type $2 \mathrm{~B}$, associated with high-grade dysplasia and superficial SMI $[8,28]$.

Kobayashi et al evaluated the diagnostic accuracy of the JNET classification using large-scale clinical practice data. Specificity, negative predictive value and accuracy were $>90 \%$ for types 1 , $2 \mathrm{~B}$ and 3 , and a sensitivity and positive predictive value $>90 \%$ were found for type $2 \mathrm{~A}$. Regarding type $2 \mathrm{~B}$ lesions, although the classification was highly specific, there was low sensitivity for the diagnosis of high-grade intramucosal neoplasia/shallow submucosal invasive cancer (42\%) [32]. As such, an additional technique, such as pit pattern assessment using chromo agents can be used to predict the depth of invasion in these lesions $[15,32,33]$. It has also been proposed that type $2 \mathrm{~B}$ should

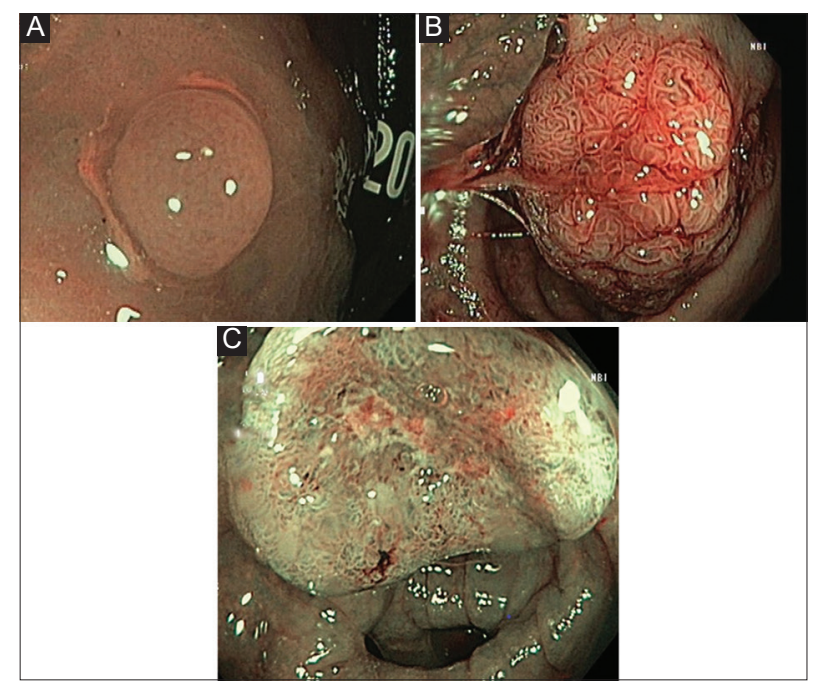

Figure 4 Narrow-band imaging (NBI) of polyps. (A) Type 1 hyperplastic polyp; (B) Type 2 - adenoma; (C) Type 3 - invasive tumor be divided into 2 subtypes, type 2B-low and type 2B-high, based on the levels of irregularity in surface and vessel patterns [34], to help optimize treatment choices. Overall, JNET can be useful in clinical practice for the characterization of colorectal lesions of types 1,2A and 3. However, one of the major pitfalls of this classification is the only fair sensitivity for the diagnosis of submucosal invasive cancer. Moreover, the JNET classification has yet to be prospectively and internationally validated. Both NICE and JNET classifications lack a sessile serrated lesion category. As sessile serrated lesions are frequently subtle and easily missed on casual inspection, and as the role of the serrated pathway becomes clearer, specific classifications of these lesions are important. A recent study has compared the accuracy of 3 different classifications for evaluating serrated lesions: NICE and JNET combined with Workgroup serrAted polypS and Polyposis (WASP) (wNICE and wJNET, respectively), and the Modified Sano's classification. The latter classification, which encompasses 5 categories, was considered to be the one that most accurately differentiates serrated and adenomatous polyps. According to the authors, the use of the Modified Sano's classification is considered beneficial as it is a "all-encompassing single classification" [35].

\section{Artificial intelligence (AI) in predicting deep SMI and LNM}

AI is currently evolving in modern gastrointestinal endoscopic technology and clinical practice. As regards its use in the differentiation between non-invasive and superficially submucosal invasive neoplasms and deeply invasive cancer, some studies have been conducted with good results. AI white light systems have demonstrated high accuracy, sensitivity and specificity in predicting the feasibility of curative endoscopic resection of large colonic lesions [36,37]. Moreover, in another study by Kudo et al [38], an AI model was built and validated in order to identify $\mathrm{T} 1$ colorectal tumors at risk for metastasis to the lymph nodes. Thus, in the near future, AI technology will be able to assist endoscopists in determining a treatment strategy, and identifying patients requiring additional surgery after endoscopic resection of T1 CRC.

\section{Histological features of T1 CRC}

After endoscopic excision of an MP, pathology has a critical role in estimating the risk of both remnant disease in the bowel wall and LNM $[18,39]$. However, pathologists may underreport on necessary elements and this may lead to inaccurate tumor risk stratification and to the potential for under- or overtreating patients $[10,11]$. A correct and complete pathologic description of MP is crucial for appropriate management (Fig. 5).

\section{Tumor differentiation}

Tumor differentiation is a subjective parameter and there are several histological grading systems for CRC [9]. To reduce the 


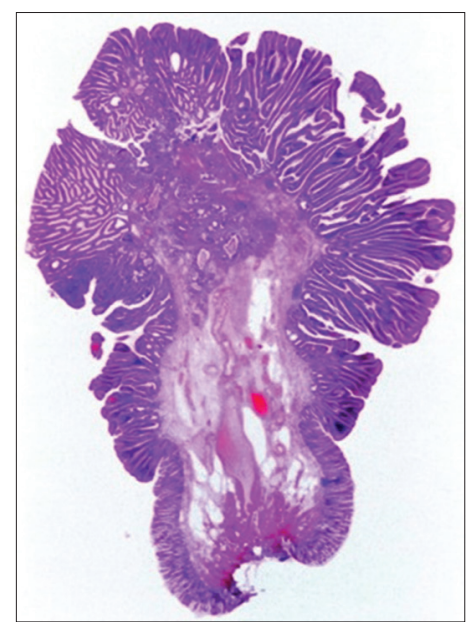

Figure 5 A malignant polyp stained with hematoxylin \& eosin $(\times 25)$. Adenocarcinoma in tubulovillous adenoma, low grade, with deep submucosal invasion $(>1000 \mu \mathrm{m})$, no evidence of lymphatic or vascular invasion, low score tumor budding, with more than $2 \mathrm{~mm}$ from tumor to cautery

interobserver variability among pathologists, a 2-grade system (well/moderate differentiation vs. poor differentiation) was proposed $[9,11]$ and adopted worldwide. Poor differentiation is an important high-risk feature in early CRC, with patients having higher rates of LNM and hematogenous metastases, and a significantly higher cancer-related mortality $[5,8,9]$.

\section{Margin of resection}

The definition of a positive margin varies in the literature, ranging from tumor at cautery to a less than $2 \mathrm{~mm}$ margin $[4,9,40]$. Traditionally, the cutoff for a positive margin was set at $2 \mathrm{~mm}$. However, over the years, scientific evidence has shown that the risk of residual disease or tumor recurrence was low when the free margin was $\geq 1 \mathrm{~mm}$. In fact, Cooper et al [41] showed that the rate of recurrence was significantly higher when the resection margin was $\leq 1 \mathrm{~mm}$ compared to a margin of $>1 \mathrm{~mm}$. Based on these results, the authors suggested a $1 \mathrm{~mm}$ margin cutoff.

More recently, there is some evidence suggesting that tumor at cautery might be an appropriate definition for a positive margin. In fact, Brown et al [39] showed that none of the MP with a margin between 0.1 and $1 \mathrm{~mm}$ had residual carcinoma in the resected surgical specimens. Berg et al [40] showed, in their cohort, that the presence of tumor at the cautery line carries a significantly higher risk of lymph node metastases when compared to a margin of greater than $0 \mathrm{~mm}$, which had no risk of residual carcinoma and minimal risk of LNM.

This potential change in the positive margin cutoff would have a significant impact on the number of patients requiring surgery, since, in the absence of other histopathological risk features, surgery would only be performed when tumor is present at the margin of resection [40]. Brown et al further proposed that cases with positive margins might also be managed by additional local resection instead of surgery, in the absence of other risk factors [39]; this is also advocated by other authors [11]. Despite this new evidence, most current international recommendations still use $\leq 1 \mathrm{~mm}$ as the definition of margin involvement [9], with the possible risk of a higher rate of unnecessary major bowel surgery.

\section{Extent of SMI}

The level of SMI in both pedunculated and sessile polyps is important in predicting the outcome of an MP. Several methodologies have been identified to evaluate the extent of SMI in MP. The Haggitt classification is a 4-level system used to assess the level of invasion in pedunculated MP [42]. Levels 1- 3 represent varying degrees of invasion into the polyp stalk, and level 4 corresponds to carcinoma invading the submucosa of underlying bowel wall below the polyp stalk, but above the muscularis propria. Haggitt reported in 1985 that patients with level 4 invasion were significantly more likely to have an adverse outcome [42]. Additionally, these polyps have a considerable risk of LNM [5]. Consequently, surgical resection is suggested for pedunculated MP which are Haggitt level 4. Kikuchi et al [43] proposed a 3-tiered system for sessile polyps, where sm 1,2, and 3 represent invasion of cancer into the first, second and deepest thirds of the submucosa, respectively. In their study, this system showed a good correlation with lymph node metastatic risk (sm1 0\%, sm 2 5\%, sm3 25\%), later confirmed by other authors [44]. The Kikuchi and Haggitt systems may be difficult to use in clinical practice, since the received sample may be fragmented or suboptimally orientated on histological sections, and because the muscularis propria, needed if the classifications are to be used, is usually not included in local endoscopic excisions [11,44].

To bypass the limitations, an alternative based on quantitative parameters has been proposed. Ueno et al [45] suggested the use of the absolute depth of invasion, which can be obtained using an optical micrometer or digital slide software on scanned slides $[8,44]$. When the invasion depth is $<1 \mathrm{~mm}$, it is considered as "superficial SMI", while an invasion depth of $\geq 1 \mathrm{~mm}$ is classified as "deep SMI", which has been associated with a considerable risk of residual disease in the bowel wall or lymph nodes after endoscopic resection [8]. Accordingly, Choi et al [46] demonstrated in a recent metaanalysis that there is a clear difference in the LNM rate between lesions with deep invasion and those with superficial invasion (OR 3.00, 95\%CI 1.36-6.62). Hence, surgical resection was recommended for MP with an SMI greater than $1000 \mu \mathrm{m}$. However, several studies have shown that the absolute depth of SMI invasion has a relatively low predictive power for LNM, especially when compared with other established risk features; for this reason, guidelines like the NCCN 2021 opt not to use it $[4,47]$.

Increasing evidence shows that the width and area of SMI may also be significant predictors of lymph node metastasis. Toh et al [48] demonstrated that an SMI width $\geq 11.5 \mathrm{~mm}$ or an invasion area $\geq 35 \mathrm{~mm}^{2}$ predicted the presence of LNM. 
However, more data are needed to better assess the importance of these 2 factors.

\section{Vascular and lymphatic Invasion}

Vascular or lymphatic invasion have been associated with the risk of LNM in many studies. The 2 factors are frequently combined into a single factor called lymphovascular invasion. Nevertheless, lymphatic invasion seems to be a more powerful predictor of LNM compared to venous invasion $[11,46]$. Bosch et al [49] included 17 studies in their systematic review and meta-analysis, showing that lymphatic invasion was the most powerful predictor of LNM (relative risk [RR] 5.2, 95\%CI 4.0-6.8), with vascular invasion being a weaker predictor ( $R R$ 2.2, 95\%CI 1.4-3.2). The combination of the 2 factors was associated with an intermediate relative risk, which is less informative $[46,49]$. Therefore, lymphatic invasion and vascular invasion should be assessed and described as separate variables to better predict LNM and aid with therapeutic decisions.

\section{Tumor budding}

Tumor budding is defined as a single tumor cell or a cell cluster of up to 4 tumor cells at the advancing front of the tumor [50]. Several studies have demonstrated the prognostic value of tumor budding $[45,46,49]$. In pT1 tumors, high tumor budding is an independent predictor of LNM. Given its prognostic value, tumor budding should be included in CRC pathology reports. In a recent International Tumor Budding Consensus Conference, a 3-tier scoring system was proposed: low (0-4 buds), intermediate (5-9 buds) and high ( $\geq 10$ buds) [50].

Currently, tumor budding reporting is recommended by the American Joint Committee on Cancer, the College of American Pathologists and the Royal College of Pathologists, although not as a core element by some of them, since the existing evidence regarding its significance in the western population is not yet considered sufficient $[8,11]$. Conversely, the Japanese Society for Cancer of the Colon and Rectum, in the 2019 guidelines for treatment of CRC, already recommends intestinal resection with lymph node dissection for MP with more than 5 tumor buds at the site of deepest invasion $[3,51]$.

\section{Management of malignant polyps}

Ultimately, the definitive diagnosis of invasive adenocarcinoma in a polyp is established by histological examination of a specimen. However, with advances in modern endoscopy and the ability to perform optical diagnosis of T1 $\mathrm{CRC}$, the management of MP begins with accurate endoscopic assessment. The recognition of specific endoscopic features will be decisive in the identification of patients who need to be referred for direct surgery, and those whose polyps are amenable to endoscopic resection.
Still, MP are more commonly diagnosed by pathologists after endoscopic resection performed without any previous suspicion of malignancy. The subsequent management will depend on risk stratification, taking into account the possibility of residual malignant cells within the bowel wall, with risk of recurrence of the disease and the risk of LNM, together with the evaluation of the patient's comorbidities and surgical risk. The decision to proceed to major surgery, consider local treatment or opt for endoscopic surveillance should be made using a multidisciplinary team approach, with input from gastroenterologists, pathologists, surgeons, oncologists and radiation oncologists, and should also involve the patient $[6,8,12,18]$.

\section{Management of MP based on endoscopic evaluation}

\section{Polyps with endoscopic features suggestive of deep SMI}

As previously discussed, NICE classification type 3 or Kudo classification type V (VN and VI) grades are highly specific for deep SMI [8]. Surface ulceration, stiffness of the lesion, fold convergence, deformity and rigidity are also predictive of deep SMI $[18,51]$. The management of polyps presenting with these features depends on their configuration. According to the latest recommendations of the US Multi-Society Task Force on Colorectal Cancer, the European Society of Gastrointestinal Endoscopy and the Japanese Society for Cancer of the Colon and Rectum [8,51,52], non-pedunculated polyps should be biopsied (in the portion of the lesion that demonstrates the most worrisome features), tattooed ( $2 \mathrm{~cm}$ distally to the lesion) and directly referred for surgery. A non-surgical approach can however be considered in case of polyps with Kudo pit pattern type VI, where endoscopic submucosal dissection (ESD) is currently accepted [53].

Pedunculated polyps, on the other hand, although having features of deep SMI, can still undergo endoscopic polypectomy since the pedunculated morphology correlates with overall favorable histological findings $[2,18]$. Therefore, en bloc endoscopic snare resection is acceptable in these cases, taking into account that the snare must be located in close proximity to the bowel wall to increase the chance of obtaining a cancer-free resection margin $[8,18,54]$. Tattooing the site is still mandatory in these cases.

\section{Polyps with endoscopic features suggestive of superficial SMI}

Superficial SMI is associated with a very low risk of residual cancer in the bowel wall or lymph nodes after endoscopic resection. Consequently, lesions with features suggestive of such a level of invasion (namely pedunculated polyps, sessile polyps NICE classification type 1 or 2, LST-NG flat elevated, LST-NG pseudo depressed, or LST-G granular homogenous and LST-G with a dominant nodule without any deep invasion features) can be managed by endoscopic resection $[8,51]$. En bloc resection is preferred over piecemeal resection, as 
histopathological assessment of complete excision is not possible and this will interfere with the prediction of LNM risk [6,12]. Moreover, piecemeal resection may also lead to high local recurrence rates (15-30\%). In a systematic review and meta-analysis by Belderbos et al [55], evaluating local recurrence after endoscopic mucosal resection (EMR) of non-pedunculated colorectal lesions, the authors found that local recurrence occurred in $20 \%$ of piecemeal resections in comparison to $3 \%$ local recurrence following en bloc resections. They also showed, in multivariate analysis, that piecemeal resection was the only independent risk factor for recurrence.

Although en bloc resection is recommended for the resection of polyps for the reasons mentioned above, there are some lesions, such as sessile or flat lesions with more than $20 \mathrm{~mm}$ diameter and favorable morphological and surface pit patterns (e.g., LST-G homogenous), that can be resected piecemeal, given their low risk of SMI [6]. Particular attention should be paid to LST-G with a dominant nodule. In these cases, the nodular area, which may contain malignant changes, should be resected en bloc, while the remaining lesion may be removed piecemeal $[8,18]$.

Whenever piecemeal resection is used, snare tip soft coagulation of the lesion's borders should be performed, as it significantly reduces the recurrence rate. In a recent multicenter randomized trial conducted by Klein et al, thermal ablation of the post-EMR mucosal defect margin resulted in a 4 -fold reduction in adenoma recurrence at first surveillance colonoscopy [56].

There are several techniques for endoscopic resection and the choice should be made according to the type of polyp and local expertise. However, if en bloc endoscopic resection is beyond the skillset of the endoscopist, patients should be referred to centers with advanced endoscopic expertise [52].

\section{Snare polypectomy}

Snare polypectomy is considered adequate for en bloc resection of pedunculated lesions presenting with features suggestive of superficial SMI [6,18]. Snare polypectomy is considered curative when the histopathology report and margins are favorable [6]. To optimize the resection margin, the snare should be placed closer to the bowel wall than to the polyp's head, particularly in case of large polyps. This positioning increases the chance of obtaining a cancer-free resection margin [54].

\section{EMR}

EMR was developed for removal of sessile polyps confined to the mucosa and submucosa. According to ESGE guidelines, to guarantee en bloc resection, EMR should be limited to lesions $\leq 20 \mathrm{~mm}$ in the colon and $\leq 25 \mathrm{~mm}$ in the rectum [52]. The recent Japanese Society for Cancer of the Colon and Rectum guidelines for the treatment of CRC, also establishes $2 \mathrm{~cm}$ as the largest size of tumor that can be easily resected en bloc by
EMR [51]. The largest study conducted to date to address EMR for early CRC reported very low rates of recurrence (0.8\%) [57].

\section{ESD}

Colorectal ESD is a technique that offers the possibility of en bloc resection of larger lesions (>20 mm), and of nongranular LST, Kudo VI pit pattern, non-lifting neoplasia, large depressed-type tumors, and carcinomas with shallow T1 (SM) invasion [53,58]. Moreover, ESD has been included in the Japanese 2019 guidelines for the treatment of CRC [51], being mainly indicated for large tumors, especially early cancers, that cannot be resected by en bloc EMR.

The method consists of lifting the submucosa by local injection, followed by submucosal dissection underneath the lesion using a special knife, to yield an en bloc specimen. The technique has obvious advantages in the treatment of MP. Outcomes of colorectal ESD have been described in the literature. In a Japanese multicenter, prospective study by Saito et al [59], the results of 1111 colorectal ESD were reported. The investigators reported en bloc and curative resection rates of $88 \%$ and $89 \%$, respectively.

However, colorectal ESD is technically difficult to perform and has a long learning curve. Training is also essential to prevent complications, namely perforations. Even in expert hands, ESD perforation rates may be as high as 5\%, while bleeding occurs in 1.5-2.2\% of cases [60]. More recently, with the development of ESD devices and the progressive establishment of ESD as a therapeutic strategy, perforation rates have been decreasing significantly, from $5 \%$ to $1.9 \%$ in recent years, particularly in Asian countries [59]. Although there is still a big difference between the Eastern and Western hemispheres in what concerns ESD development and implementation in daily practice, experience with ESD is accumulating in the West [6]. By enabling en bloc resection of selected early CRCs, ESD offers high rates of curative resection, obviating the need for surgery and subsequently lowering overall costs and improving morbimortality [60].

\section{Endoscopic full-thickness resection (EFTR)}

EFTR is a novel treatment for colorectal lesions not amenable to conventional endoscopic resection (e.g., EMR and ESD) because of the inherent risks of perforation and the technical difficulty [61]. The full-thickness resection device (FTRD) allows clip-assisted EFTR with a single-step technique. It is effective in the resection of difficult to-resect colorectal lesions $\leq 2 \mathrm{~cm}$, namely lesions with no-lifting sign (either recurrent lesions or previously untreated lesions), or at difficult anatomic locations (such as lesions at a diverticulum and the appendiceal orifice) $[62,63]$.

In the studies published to date, FTRD was also used to treat a subgroup of patients with early carcinomas. A recent multicenter, retrospective study conducted by Kuellmer et al [64], evaluated the efficacy, safety, and clinical 
value of EFTR for early CRC. One hundred fifty-six patients were involved, the main indications being incompletely resected malignant polyps and non-lifting malignant colorectal lesions without prior attempt at resection. Technical success was $92.3 \%$, with an R0 resection rate of $71.8 \%$, this being significantly inferior in the group of non-lifting lesions (60.9\%) in comparison to the groups with repeat resection of MP (87.5\%). Based on their results, the authors consider that EFTR for early CRC is a feasible, effective and safe technique. It allows exact histological risk stratification and can avoid surgery for low-risk lesions. Nevertheless, prospective studies are required to further define indications for EFTR in malignant colorectal lesions and to evaluate long-term outcomes.

\section{Management of MP after endoscopic resection}

As previously stated, the most common scenario regarding MP is a patient presenting for evaluation after a resected polyp, thought to have a benign appearance on endoscopy, is found to have an invasive focus of adenocarcinoma in the final pathology report. The management of MP following endoscopic removal is difficult, since it depends on both the risk of residual or recurrent disease and of LNM, and the patients' surgical risk $[2,6,65]$.

Based on the morphological, endoscopic and histological prognostic features detailed previously, MP should be stratified into high- and low-risk polyps. Generally, high-risk polyps are further submitted to major surgical resection, whereas low-risk polyps are managed adequately with appropriate surveillance. In cases where the risk of residual cancer and the risk of surgical mortality is similar, patients' opinions are even more important. The recommendations of the US Multi-Society Task Force on Colorectal Cancer for the management of MP emphasize shared decision-making, since the overall mortality after colon cancer surgery is $1-8 \%$ and correlates with patient age and comorbidities [8].

For convenience, polyps of the colon and rectum are considered together in the literature. However, there are differences between the 2 sites regarding the availability of techniques to locally excise the polyps, the possibility of using radiotherapy, and the surgical morbimortality. In the present review, we will differentiate between the management of MP located in the colon or the rectum, following endoscopic resection. Another important difference that should be taken into account in the management of MP is polyp morphology, since the risk of incomplete endoscopic resection and LNM is much lower in pedunculated than in non-pedunculated T1 CRC [66].

\section{MP of the colon}

Patients with MP of the colon with high-risk featuresnamely poor differentiation, presence of lymphovascular invasion, deep SMI $(>1 \mathrm{~mm})$, positive margin, piecemeal resection or tumor budding-are usually recommended to undergo surgical resection with lymph node clearance, if medically fit $[2,6,8,12,65]$. Regarding pedunculated MP, Backes et al [66] found that lymphovascular invasion, Haggitt level 4 invasion, muscularis mucosae type B (incompletely or completely disrupted), poorly differentiated clusters and tumor budding, were the histologic factors indicating the need for surgery. Based on these findings, they developed a prediction model to better estimate the indication for surgery in pedunculated $\mathrm{T} 1 \mathrm{CRC}$.

Nevertheless, it is worth saying that, even in high-risk MP, a high proportion of patients do not have evidence of residual disease in the bowel wall, nor in the draining lymph nodes. In the Brisbane series of $239 \mathrm{MP}, 140$ were submitted to subsequent surgical resection because of the presence of high-risk features. Pathological analysis of the resected specimens revealed the presence of residual disease in 20 cases (8.4\%), including 12 cases of nodal metastasis and 9 cases of residual carcinoma in the large bowel wall [39]. Based on their results, the authors suggest that surgical resection should be recommended only if 2 or more adverse histological features are present (risk of nodal metastasis: $23.3 \%$ ). In cases where only one risk feature is present, the authors estimated a $4.5 \%$ risk of nodal metastasis, thus suggesting that the surgical option should be weighed against the patients' comorbidities, also taking into account tumor location.

On the other hand, when an MP of the colon is considered to be a low-risk polyp, with none of the poor prognostic features, endoscopic resection is considered curative $[4,6,12,65]$. The patient is therefore included in a surveillance program, undergoing regular colonoscopies to detect early recurrence and minimize the risk of metachronous disease. Currently, there is no consensus regarding the timing for endoscopic follow up, since evidence is limited [4,9]. Most authors suggest that the first surveillance colonoscopy be performed 3-6 months after endoscopic removal of the MP, with the subsequent evaluations being scheduled according to risk and findings $[6,8,12,21,65]$.

Radiological assessment of residual bowel wall disease, lymph node and distant metastasis is controversial in the case of colonic MP. As the sensitivity and specificity of computed tomography (CT) scanning is considered insufficient for the detection of residual disease in the bowel wall or lymph nodes after endoscopic resection of MP, it should not be used for surgical decision making. For the same reasons, radiological surveillance is not recommended $[6,9,18]$. However, the position statement of the Association of Coloproctology of Great Britain and Ireland recommends a baseline abdominal pelvic and chest CT scan 3-4 weeks following endoscopic resection [6]. The objective of this $\mathrm{CT}$ is to have a baseline comparator for subsequent evaluation in case of future suspected recurrent disease.

\section{MP of the rectum}

Decisions regarding the management of rectal MP are particularly challenging. On the one hand, rectal cancers 
harbor a higher risk of local recurrence compared to colonic cancers, independently of stage. On the other hand, the morbidity of rectal surgery is higher than that of colonic surgery, and includes the possibility of developing sexual dysfunction, urinary disturbances and low anterior rectal syndrome [6,18]. Moreover, for very low rectal lesions, the appropriate oncological surgery implies a permanent colostomy. The risk of residual disease and LNM should, therefore, be even more thoroughly weighed against the risks of surgery when determining the management of rectal MP following endoscopic resection.

Regarding rectal MP without poor prognostic features after en bloc endoscopic resection, surveillance is recommended, as for colonic MP. In these cases, no further imaging assessment is recommended, nor imaging surveillance. Patients with rectal MP presenting with criteria of a non-curative endoscopic resection-fragmented specimen or unfavorable histological features like third submucosal layer (sm3) invasion, positive margin, poorly differentiated, lymphovascular invasionshould be referred for definitive treatment, which encompasses major surgery in the majority of cases $[47,67]$. Before surgery, distant and local staging should be performed, and should include a chest CT, an abdominal CT or magnetic resonance imaging (MRI) and a pelvic MRI or, if MRI is contraindicated, pelvic CT plus endorectal ultrasound [47].

Regarding the surgical procedure, different strategies are recommended, namely abdominoperineal resection or low anterior resection, with total mesorectal excision, depending on the distal margin of the tumor. Transanal local excision can also be considered, as an alternative to radical surgery, for patients with a high surgical risk, and always after discussion with the patient. Transanal local excision, which includes traditional transanal excision, and more advanced techniques (transanal endoscopic microsurgery and transanal minimally invasive surgery), allows for en bloc local excision of rectal tumors, without compromising anorectal function. Morbidity is therefore substantial lower [21,47]. However, since lymph node resection is not performed, oncological outcomes may be compromised in patients with high-risk MP [68]. The only scenario where local excision using transanal surgery may be a safe option is when a rectal MP presents with an inadequately clear margin after endoscopic resection, but without any other risk features [12].

Another possible approach in the management of low rectal MP presenting with high-risk features after endoscopic resection is the use of chemoradiotherapy, followed by re-staging, with the aim of using a "watch-and-wait" (W\&W) strategy. In their most recent guidelines, both the European Society for Medical Oncology and the National Comprehensive Cancer Network (NCCN) consider the W\&W approach for rectal cancer $[47,67]$. This strategy is indicated for the treatment of locally advanced rectal cancer and consists of omitting surgery after chemoradiation when a complete clinical response is achieved. The patient is then integrated into a strict surveillance program, with surgery being performed in case of tumor regrowth [47]. Despite promising results, with good oncological outcomes, available evidence is still considered by many societies to be insufficient and not robust enough to support W\&W as the standard approach in clinical practice $[47,67,69]$. Nevertheless, the NCCN guidelines, for instance, consider W\&W protocols may be used in centers with experienced multidisciplinary teams and after careful discussion with the patients [47]. Regarding W\&W in the management of low rectal MP with high-risk features, aiming for organ preservation, limited data are available. The few studies that have included patients with early-stage tumors [69] provide interesting results, favoring the use of chemoradiation followed by surveillance in these patients. The currently ongoing STAR-TREC trial will bring new evidence regarding this issue [70].

\section{Concluding remarks}

MP are increasingly found along with the widespread use of colonoscopy. Careful endoscopic inspection of colorectal polyps is essential to distinguish between MP amenable for endoscopic resection and those requiring major oncological surgery. Endoscopists should be capable of performing an optical diagnosis through the use of image enhancing techniques and should have a profound knowledge about the endoscopic resection techniques best suited for each type of polyp. Pathological reports are then essential for further management of MP. The presence of high-risk features (positive margin, deep SMI (>1 mm), poorly differentiated carcinoma, lymphovascular invasion, tumor budding and piecemeal resection) indicates a need for surgery in the majority of cases, although location, comorbidities and patient preference should be taken into account when making the final decision.

\section{References}

1. Dekker E, Tanis PJ, Vleugels JLA, Kasi PM, Wallace MB. Colorectal cancer. Lancet 2019;394:1467-1480.

2. Labianca R, Nordlinger B, Beretta GD, et al. ESMO Guidelines Working Group. Early colon cancer: ESMO Clinical Practice Guidelines for diagnosis, treatment and follow-up. Ann Oncol 2013;24 Suppl 6:vi64-vi72.

3. Watanabe T, Itabashi M, Shimada Y, et al; Japanese Society for Cancer of the Colon and Rectum. Japanese Society for Cancer of the Colon and Rectum (JSCCR) guidelines 2010 for the treatment of colorectal cancer. Int J Clin Oncol 2012;17:1-29.

4. National Comprehensive Cancer Network. Colon Cancer (Version 2.2021). Available from: https://www.nccn.org/guidelines/guidelinesdetail? category $=1 \& \mathrm{id}=1428$ [Accessed 18 November 2021].

5. Kuo E, Wang K, Liu X. A focused review on advances in risk stratification of malignant polyps. Gastroenterology Res 2020;13:163-183.

6. Williams JG, Pullan RD, Hill J, et al; Association of Coloproctology of Great Britain and Ireland. Management of the malignant colorectal polyp: ACPGBI position statement. Colorectal Dis 2013;15 Suppl 2:1-38.

7. Bettington $\mathrm{M}$, Walker $\mathrm{N}$, Clouston A, Brown I, Leggett B, Whitehall V. The serrated pathway to colorectal carcinoma: current concepts and challenges. Histopathology 2013;62:367-386.

8. Shaukat A, Kaltenbach T, Dominitz JA, et al. Endoscopic 
recognition and management strategies for malignant colorectal polyps: recommendations of the US multi-society task force on colorectal cancer. Gastroenterology 2020;159:1916-1934.

9. Teo NZ, Wijaya R, Ngu JC. Management of malignant colonic polyps. J Gastrointest Oncol 2020;11:469-474.

10. Gimon TI, Dykstra MA, Chezar K, Buie WD, MacLean A. Malignant colorectal polyp pathology: are we getting sufficient information to make decisions? Dis Colon Rectum 2020;63:135-142.

11. Brockmoeller SF, West NP. Predicting systemic spread in early colorectal cancer: Can we do better? World J Gastroenterol 2019;25:2887-2897.

12. Cancer council Australia. Guidelines: Colorectal cancer/ Colonoscopy surveillance/Malignant polyps. Available from: https://wiki.cancer.org.au/australia/Guidelines: Colorectal_ cancer/Colonoscopy_surveillance/Malignant_polyps [Accessed 18 November 2021].

13. Nascimbeni R, Burgart LJ, Nivatvongs S, Larson DR. Risk of lymph node metastasis in T1 carcinoma of the colon and rectum. Dis Colon Rectum 2002;45:200-206.

14. Puig I, López-Cerón M, Arnau A, et al; EndoCAR group, Spanish Gastroenterological Association and the Spanish Digestive Endoscopy Society. Accuracy of the narrow-band imaging international colorectal endoscopic classification system in identification of deep invasion in colorectal polyps. Gastroenterology 2019;156:75-87.

15. Puig I, Mármol C, Bustamante M. Endoscopic imaging techniques for detecting early colorectal cancer. Curr Opin Gastroenterol 2019;35:432-439.

16. Backes Y, Moss A, Reitsma JB, Siersema PD, Moons LM. Narrow band imaging, magnifying chromoendoscopy, and gross morphological features for the optical diagnosis of T1 colorectal cancer and deep submucosal invasion: a systematic review and meta-analysis. Am J Gastroenterol 2017;112:54-64.

17. Backes Y, Schwartz MP, Ter Borg F, et al; Dutch T1 CRC Working Group. Multicentre prospective evaluation of real-time optical diagnosis of $\mathrm{T} 1$ colorectal cancer in large non-pedunculated colorectal polyps using narrow band imaging (the OPTICAL study). Gut 2019;68:271-279.

18. Rex DK, Shaukat A, Wallace MB. Optimal management of malignant polyps, from endoscopic assessment and resection to decisions about surgery. Clin Gastroenterol Hepatol 2019;17:1428-1437.

19. Hewett DG, Kaltenbach T, Sano Y, et al. Validation of a simple classification system for endoscopic diagnosis of small colorectal polyps using narrow-band imaging. Gastroenterology 2012;143:599-607.

20. The Paris endoscopic classification of superficial neoplastic lesions: esophagus, stomach, and colon: November 30 to December 1, 2002. Gastrointest Endosc 2003;58:S3-S43.

21. Bartel MJ, Brahmbhatt BS, Wallace MB. Management of colorectal T1 carcinoma treated by endoscopic resection from the Western perspective. Dig Endosc 2016;28:330-341.

22. Bogie RMM, Veldman MHJ, Snijders LARS, et al. Endoscopic subtypes of colorectal laterally spreading tumors (LSTs) and the risk of submucosal invasion: a meta-analysis. Endoscopy 2018;50:263-282.

23. Uraoka T, Saito Y, Matsuda T, et al. Endoscopic indications for endoscopic mucosal resection of laterally spreading tumours in the colorectum. Gut 2006;55:1592-1597.

24. Shahidi N, Vosko S, van Hattem WA, Sidhu M, Bourke MJ. Optical evaluation: the crux for effective management of colorectal neoplasia. Therap Adv Gastroenterol 2020;13:1756284820922746.

25. Burgess NG, Hourigan LF, Zanati SA, et al. Risk stratification for covert invasive cancer among patients referred for colonic endoscopic mucosal resection: a large multicenter cohort. Gastroenterology 2017;153:732-742.

26. Matsuda T, Saito Y, Fujii T, et al. Size does not determine the grade of malignancy of early invasive colorectal cancer. World $J$ Gastroenterol 2009;15:2708-2713.

27. Bisschops R, East JE, Hassan C, et al. Advanced imaging for detection and differentiation of colorectal neoplasia: European Society of Gastrointestinal Endoscopy (ESGE) Guideline - Update 2019. Endoscopy 2019;51:1155-1179.

28. East JE, Vleugels JL, Roelandt P, et al. Advanced endoscopic imaging: European Society of Gastrointestinal Endoscopy (ESGE) Technology Review. Endoscopy 2016;48:1029-1045.

29. Togashi K, Nemoto D, Utano K, et al. Blue laser imaging endoscopy system for the early detection and characterization of colorectal lesions: a guide for the endoscopist. Therap Adv Gastroenterol 2016;9:50-56.

30. Hayashi N, Tanaka S, Hewett DG, et al. Endoscopic prediction of deep submucosal invasive carcinoma: validation of the narrowband imaging international colorectal endoscopic (NICE) classification. Gastrointest Endosc 2013;78:625-632.

31. Sano Y, Tanaka S, Kudo SE, et al. Narrow-band imaging (NBI) magnifying endoscopic classification of colorectal tumors proposed by the Japan NBI Expert Team. Dig Endosc 2016;28:526-533.

32. Kobayashi S, Yamada M, Takamaru H, et al. Diagnostic yield of the Japan NBI Expert Team (JNET) classification for endoscopic diagnosis of superficial colorectal neoplasms in a large-scale clinical practice database. United European Gastroenterol J 2019;7:914-923.

33. Sumimoto K, Tanaka S, Shigita K, et al. Clinical impact and characteristics of the narrow-band imaging magnifying endoscopic classification of colorectal tumors proposed by the Japan NBI Expert Team. Gastrointest Endosc 2017;85:816-821.

34. Sumimoto K, Tanaka S, Shigita K, et al. Diagnostic performance of Japan NBI Expert Team classification for differentiation among noninvasive, superficially invasive, and deeply invasive colorectal neoplasia. Gastrointest Endosc 2017;86:700-709.

35. Zorron Cheng Tao $\mathrm{Pu} \mathrm{L}$, Yamamura T, Nakamura $\mathrm{M}$, et al. Comparison of different virtual chromoendoscopy classification systems for the characterization of colorectal lesions. JGH Open 2020;4:818-826.

36. Lui TKL, Wong KKY, Mak LLY, Ko MKL, Tsao SKK, Leung WK. Endoscopic prediction of deeply submucosal invasive carcinoma with use of artificial intelligence. Endosc Int Open 2019;7:E514-E520.

37. Luo X, Wang J, Han Z, et al. Artificial intelligence-enhanced whitelight colonoscopy with attention guidance predicts colorectal cancer invasion depth. Gastrointest Endosc 2021;94:627-638.

38. Kudo SE, Ichimasa K, Villard B, et al. Artificial intelligence system to determine risk of $\mathrm{T} 1$ colorectal cancer metastasis to lymph node. Gastroenterology 2021;160:1075-1084.

39. Brown IS, Bettington ML, Bettington A, Miller G, Rosty C. Adverse histological features in malignant colorectal polyps: a contemporary series of 239 cases. J Clin Pathol 2016;69:292-299.

40. Berg KB, Telford JJ, Gentile L, Schaeffer DF. Re-examining the 1-mm margin and submucosal depth of invasion: a review of 216 malignant colorectal polyps. Virchows Arch 2020;476:863-870.

41. Cooper HS, Deppisch LM, Gourley WK, et al. Endoscopically removed malignant colorectal polyps: clinicopathologic correlations. Gastroenterology 1995;108:1657-1665.

42. Haggitt RC, Glotzbach RE, Soffer EE, Wruble LD. Prognostic factors in colorectal carcinomas arising in adenomas: implications for lesions removed by endoscopic polypectomy. Gastroenterology 1985;89:328-336.

43. Kikuchi R, Takano M, Takagi K, et al. Management of early invasive colorectal cancer. Risk of recurrence and clinical guidelines. Dis Colon Rectum 1995;38:1286-1295.

44. Hagen CE, Farooq A. Histologic evaluation of malignant polyps and low-stage colorectal carcinoma. Arch Pathol Lab Med 2019;143:1450-1454 
45. Ueno $\mathrm{H}$, Mochizuki $\mathrm{H}$, Hashiguchi $\mathrm{Y}$, et al. Risk factors for an adverse outcome in early invasive colorectal carcinoma. Gastroenterology 2004;127:385-394.

46. Choi JY, Jung SA, Shim KN, et al; Korean ESD Study Group. Metaanalysis of predictive clinicopathologic factors for lymph node metastasis in patients with early colorectal carcinoma. $J$ Korean Med Sci 2015;30:398-406.

47. National Comprehensive Cancer Network. Rectal Cancer (Version 1.2021). Available from: https://www.nccn.org/guidelines/ guidelines-detail?category=1\&id=1461 [Accessed 18 November 2021].

48. Toh EW, Brown P, Morris E, Botterill I, Quirke P. Area of submucosal invasion and width of invasion predicts lymph node metastasis in pT1 colorectal cancers. Dis Colon Rectum 2015;58:393-400.

49. Bosch SL, Teerenstra S, de Wilt JH, Cunningham C, Nagtegaal ID. Predicting lymph node metastasis in pT1 colorectal cancer: a systematic review of risk factors providing rationale for therapy decisions. Endoscopy 2013;45:827-834.

50. Lugli A, Kirsch R, Ajioka Y, et al. Recommendations for reporting tumor budding in colorectal cancer based on the International Tumor Budding Consensus Conference (ITBCC) 2016. Mod Pathol 2017;30:1299-1311.

51. Hashiguchi Y, Muro K, Saito Y, et al; Japanese Society for Cancer of the Colon and Rectum. Japanese Society for Cancer of the Colon and Rectum (JSCCR) guidelines 2019 for the treatment of colorectal cancer. Int J Clin Oncol 2020;25:1-42.

52. Ferlitsch M, Moss A, Hassan C, et al. Colorectal polypectomy and endoscopic mucosal resection (EMR): European Society of Gastrointestinal Endoscopy (ESGE) Clinical Guideline. Endoscopy 2017;49:270-297.

53. Tanaka S, Kashida H, Saito Y, et al. Japan Gastroenterological Endoscopy Society guidelines for colorectal endoscopic submucosal dissection/endoscopic mucosal resection. Dig Endosc 2020;32:219-239.

54. Ciocalteu A, Gheonea DI, Saftoiu A, Streba L, Dragoescu NA, Tenea-Cojan TS. Current strategies for malignant pedunculated colorectal polyps. World J Gastrointest Oncol 2018;10:465-475.

55. Belderbos TD, Leenders M, Moons LM, Siersema PD. Local recurrence after endoscopic mucosal resection of nonpedunculated colorectal lesions: systematic review and meta-analysis. Endoscopy 2014;46:388-402.

56. Klein A, Tate DJ, Jayasekeran V, et al. Thermal ablation of mucosal defect margins reduces adenoma recurrence after colonic endoscopic mucosal resection. Gastroenterology 2019;156:604-613.

57. Park JJ, Cheon JH, Kwon JE, et al. Clinical outcomes and factors related to resectability and curability of EMR for early colorectal cancer. Gastrointest Endosc 2011;74:1337-1346.

58. Pimentel-Nunes P, Dinis-Ribeiro M, Ponchon T, et al. Endoscopic submucosal dissection: European Society of Gastrointestinal Endoscopy (ESGE) Guideline. Endoscopy 2015;47:829-854.

59. Saito Y, Yamada M, So E, et al. Colorectal endoscopic submucosal dissection: Technical advantages compared to endoscopic mucosal resection and minimally invasive surgery. Dig Endosc 2014;26(Suppl 1):52-61.

60. Draganov PV, Wang AY, Othman MO, Fukami N. AGA Institute clinical practice update: endoscopic submucosal dissection in the United States. Clin Gastroenterol Hepatol 2019;17:16-25.

61. Schmidt A, Bauerfeind P, Gubler C, Damm M, Bauder M, Caca K. Endoscopic full-thickness resection in the colorectum with a novel over-the-scope device: first experience. Endoscopy 2015;47:719-725.

62. Schmidt A, Beyna T, Schumacher B, et al. Colonoscopic fullthickness resection using an over-the-scope device: a prospective multicentre study in various indications. Gut 2018;67:1280-1289.

63. Meier B, Stritzke B, Kuellmer A, et al. Efficacy and safety of endoscopic full-thickness resection in the colorectum: results from the German Colonic FTRD Registry. Am J Gastroenterol 2020;115:1998-2006.

64. Kuellmer A, Mueller J, Caca K, et al; FTRD study group. Endoscopic full-thickness resection for early colorectal cancer. Gastrointest Endosc 2019;89:1180-1189.

65. Aarons CB, Shanmugan S, Bleier JI. Management of malignant colon polyps: current status and controversies. World J Gastroenterol 2014;20:16178-16183.

66. Backes Y, Elias SG, Groen JN, et al; Dutch T1 CRC Working Group. Histologic factors associated with need for surgery in patients with pedunculated T1 colorectal carcinomas. Gastroenterology 2018;154:1647-1659.

67. Glynne-Jones R, Wyrwicz L, Tiret E, et al; ESMO Guidelines Committee. Rectal cancer: ESMO Clinical Practice Guidelines for diagnosis, treatment and follow-up. Ann Oncol 2018;29:iv263.

68. Althumairi AA, Gearhart SL. Local excision for early rectal cancer: transanal endoscopic microsurgery and beyond. J Gastrointest Oncol 2015;6:296-306.

69. Bernier L, Balyasnikova S, Tait D, Brown G. Watch-and-wait as a therapeutic strategy in rectal cancer. Curr Colorectal Cancer Rep 2018;14:37-55.

70. Rombouts AJM, Al-Najami I, Abbott NL, et al; for STAR-TREC Collaborative Group. Can we $S$ ave the rectum by watchful waiting or $\mathrm{T}$ rans A nal microsurgery following (chemo) $\mathrm{R}$ adiotherapy versus $\mathrm{T}$ otal mesorectal excision for early RE ctal $\mathrm{C}$ ancer (STARTREC study)?: protocol for a multicentre, randomised feasibility study. BMJ Open 2017;7:e019474. 